\title{
DUALITY AND SERRE FUNCTOR IN HOMOTOPY CATEGORIES
}

\author{
J. ASADOLLAHI, N. ASADOLLAHI, R. HAFEZI AND R. VAHED
}

\begin{abstract}
For a (right and left) coherent ring $A$, we show that there exists a duality between homotopy categories $\mathbb{K}^{\mathrm{b}}\left(\bmod -A^{\mathrm{op}}\right)$ and $\mathbb{K}^{\mathrm{b}}(\bmod -A)$. If $A=\Lambda$ is an artin algebra of finite global dimension, this duality restricts to a duality between their subcategories of acyclic complexes, $\mathbb{K}_{\mathrm{ac}}^{\mathrm{b}}\left(\bmod -\Lambda^{\mathrm{op}}\right)$ and $\mathbb{K}_{\mathrm{ac}}^{\mathrm{b}}(\bmod -\Lambda)$. As a result, it will be shown that, in this case, $\mathbb{K}_{\mathrm{ac}}^{\mathrm{b}}(\bmod -\Lambda)$ admits a Serre functor and hence has Auslander-Reiten triangles.
\end{abstract}

\section{INTROdUCTION}

A contravariant functor between two categories that is an equivalence is called a duality. The role and importance of dualities is known in representation theory of algebras. Let $A$ be a right and left coherent ring. In this paper, we introduce and study a duality between the bounded homotopy categories of finitely generated right and finitely generated left $A$-modules, denoted by $\mathbb{K}^{\mathrm{b}}(\bmod -A)$ and $\mathbb{K}^{\mathrm{b}}\left(\bmod -A^{\mathrm{op}}\right)$, respectively. We gain this duality starting from an equivalence

$$
\mu: \mathbb{D}\left(\operatorname{Mod}-\left(\bmod -A^{\mathrm{op}}\right)\right) \longrightarrow \mathbb{D}\left(\operatorname{Mod}-(\bmod -A)^{\mathrm{op}}\right)
$$

of derived categories of functor categories.

The relationship between $\mu$ and some known dualities will be discussed. In particular, it is shown that, Proposition 3.4 below, there is a close relationship between $\mu$ and the Auslander-Gruson-Jensen duality

$$
\mathfrak{D}: \bmod -\left(\bmod -A^{\mathrm{op}}\right)^{\mathrm{op}} \longrightarrow \bmod -(\bmod -A)^{\mathrm{op}} \text {. }
$$

Let $\Lambda$ be an artin algebra of finite global dimension over a commutative artinian ring $R$. We show that in this case, the above duality between $\mathbb{K}^{\mathrm{b}}\left(\bmod -\Lambda^{\mathrm{op}}\right)$ and $\mathbb{K}^{\mathrm{b}}(\bmod -\Lambda)$ restricts to a duality between $\mathbb{K}_{\mathrm{ac}}^{\mathrm{b}}\left(\bmod -\Lambda^{\mathrm{op}}\right)$ and $\mathbb{K}_{\mathrm{ac}}^{\mathrm{b}}(\bmod -\Lambda)$, where for an abelian category $\mathcal{A}, \mathbb{K}_{\mathrm{ac}}^{\mathrm{b}}(\mathcal{A})$ is the full subcategory of $\mathbb{K}^{\mathrm{b}}(\mathcal{A})$ consisting of all acyclic complexes. This, in turn, implies that there is an equivalence of triangulated categories

$$
\frac{\mathbb{K}^{\mathrm{b}}(\bmod -\Lambda)}{\mathbb{K}^{\mathrm{b}}(\operatorname{prj}-\Lambda)} \stackrel{\sim}{\longrightarrow} \frac{\mathbb{K}^{\mathrm{b}}(\bmod -\Lambda)}{\mathbb{K}^{\mathrm{b}}(\operatorname{inj}-\Lambda)}
$$

Note that under certain conditions, the quotient $\frac{\mathbb{K}^{\mathrm{b}}(\bmod -\Lambda)}{\mathbb{K}^{\mathrm{b}}(\operatorname{prj}-\Lambda)}$ is equivalent to the relative singularity category introduced and studied recently in [KY], see Remark 3.8.

Finally, we show that $\mathbb{K}_{\mathrm{ac}}^{\mathrm{b}}(\bmod -\Lambda)$ admits a Serre functor $\mathbb{S}$ in the sense of $[\mathrm{BK}]$. By a well-known result of Reiten and Van den Bergh [RV, Theorem I.2.4], the existence of a Serre functor is equivalent to the existence of Auslander-Reiten triangles in a category and so we deduce that $\mathbb{K}_{\mathrm{ac}}^{\mathrm{b}}(\bmod -\Lambda)$ admits Auslander-Reiten triangles.

2010 Mathematics Subject Classification. 18E30, 16E35, $18 \mathrm{G} 25$.

Key words and phrases. Functor category, derived category, artin algebra, duality. 


\section{Preliminaries}

Throughout the paper, $A$ denotes a right and left coherent ring. $A$-module means right $A$ module. Mod- $A$, resp. $\bmod -A$, denotes the category of $A$-modules, resp. finitely presented $A$ modules. Prj- $A$, resp. prj- $A$, denotes the full subcategory of Mod- $A$, resp. mod- $A$, consisting of projective $A$-modules. Inj- $A$ and inj- $A$ represent injectives and finitely presented injectives, resp. For an additive category $\mathcal{A}, \mathbb{D}(\mathcal{A})$, resp. $\mathbb{K}(\mathcal{A})$, denotes the derived category, resp. homotopy category, of $\mathcal{A}$. As usual, the bounded derived, resp. homotopy, category of $\mathcal{A}$, will be denoted by $\mathbb{D}^{\mathrm{b}}(\mathcal{A})$, resp. $\mathbb{K}^{\mathrm{b}}(\mathcal{A})$.

2.1. Following Auslander we let $\operatorname{Mod}-(\bmod -A), \operatorname{resp} . \operatorname{Mod}-(\bmod -A)^{\mathrm{op}}$, denote the category of all contravariant, resp. covariant, additive functors from mod- $A$ to $\mathcal{A} b$, the category of abelian groups. Throughout we shall use parenthesis to denote the Hom sets. An object $F$ of $\operatorname{Mod}-(\bmod -A), \operatorname{resp} . \operatorname{Mod}-(\bmod -A)^{\mathrm{op}}$, is called coherent if there exists a short exact sequence

$$
\begin{aligned}
&(-, X) \longrightarrow(-, Y) \longrightarrow F \longrightarrow 0, \\
& \text { resp. }(X,-) \longrightarrow(Y,-) \longrightarrow F \longrightarrow 0,
\end{aligned}
$$

of functors, where $X$ and $Y$ belong to $\bmod -A$. We let $\bmod -(\bmod -A), \operatorname{resp} . \bmod -(\bmod -A)^{\mathrm{op}}$, denote the full subcategory of $\operatorname{Mod}-(\bmod -A)$, resp. Mod- $(\bmod -A)^{\mathrm{op}}$, consisting of all coherent functors. It is known $[\mathrm{A} 1]$ that both $\operatorname{Mod}-(\bmod -A)$ and $\bmod -(\bmod -A)$ and also their counterparts of covariant functors are abelian categories with enough projective objects.

Special objects of such categories have been studied by several authors. In particular, an object $F$ of Mod-(mod- $A)$ is flat if and only if there exists an $A$-module $M$ such that $F \cong \operatorname{Hom}_{A}(-, M)$, see [JL, Theorem B.10]. We let $\mathcal{F}(\bmod -A)$ denote the full subcategory of Mod- $(\bmod -A)$ consisting of all flat functors.

2.2. A sequence $0 \rightarrow X \rightarrow Y \rightarrow Z \rightarrow 0$ of $A$-modules is called pure exact if the induced sequence

$$
0 \longrightarrow X \otimes_{A} M \longrightarrow Y \otimes_{A} M \longrightarrow Z \otimes_{A} M \longrightarrow 0,
$$

is exact, for every left $A$-module $M$. A module $P$ is called pure projective, resp. pure injective, if it is projective, resp. injective, with respect to the class of all pure exact sequences. We let $\operatorname{PPrj}-A$, resp. PInj- $A$, denote the full subcategory of Mod- $A$ consisting of all pure projective, resp. pure injective, $A$-modules.

The derived category of $A$ with respect to the pure exact structure is called pure derived category and is denoted by $\mathbb{D}_{\text {pur }}(\operatorname{Mod}-A)$. Krause $[\mathrm{K} 1]$ introduced and studied this category in deep. He [K1, Corollary 6] proved that for a ring $A$, there exists a triangle equivalence

$$
\mathbb{D}_{\text {pur }}(\operatorname{Mod}-A) \simeq \mathbb{D}(\operatorname{Mod}-(\bmod -A)) \text {. }
$$

2.3. Let $\mathbb{K}_{\mathrm{ac}}(\mathcal{F}(\bmod -A))$ be the full subcategory of $\mathbb{K}(\mathcal{F}(\bmod -A))$ formed by all acyclic complexes of flat functors. It is a thick subcategory of $\mathbb{K}(\mathcal{F}(\bmod -A))$ and so we have the quotient category $\frac{\mathbb{K}(\mathcal{F}(\bmod -A))}{\mathbb{K}_{\mathrm{ac}}(\mathcal{F}(\bmod -A))}$. By $[\mathrm{AAHV}$, Lemma 4.4], there exists a triangle equivalence

$$
\varphi: \mathbb{D}(\operatorname{Mod}-(\bmod -A)) \longrightarrow \frac{\mathbb{K}(\mathcal{F}(\bmod -A))}{\mathbb{K}_{\mathrm{ac}}(\mathcal{F}(\bmod -A))} .
$$

This equivalence maps every complex $\mathbf{X}$ in $\mathbb{D}(\operatorname{Mod}-(\bmod -A))$ to a complex $\mathbf{F}$ in $\mathbb{K}(\mathcal{F}(\bmod -A))$, where $\mathbf{F}$ fits into a short exact sequence

$$
0 \longrightarrow \mathbf{C} \longrightarrow \mathbf{F} \longrightarrow \mathbf{X} \longrightarrow 0
$$

in $\mathbb{C}(\operatorname{Mod}-(\bmod -A))$ in which $\operatorname{Ext}^{i}\left(\mathbf{F}^{\prime}, \mathbf{C}\right)=0$, for $i>0$ and for all $\mathbf{F}^{\prime} \in \mathbb{C}(\mathcal{F}(\bmod -A))$. 
2.4. Recall that an object $C$ of an abelian category $\mathcal{A}$ is called cotorsion if for every flat object $F, \operatorname{Ext}^{1}(F, C)=0$. Let Cot- $\mathcal{F}(\bmod -A)$ denote the full subcategory of $\mathcal{F}(\bmod -A)$ consisting of all cotorsion-flat functors. By $[\mathrm{H}$, Theorem 4$]$, a flat functor $(-, M)$ in $\operatorname{Mod}-(\bmod -A)$ is cotorsion if and only if $M$ is a pure-injective module. So the fully faithful functor $U$ : Mod- $A \longrightarrow \operatorname{Mod}-(\bmod -A)$ induces an equivalence

$$
\mathbb{K}(\mathrm{PInj}-A) \stackrel{\mathbb{K}(U)}{\longrightarrow} \mathbb{K}(\operatorname{Cot}-\mathcal{F}(\bmod -A)),
$$

of triangulated categories.

2.5. By Remark 4.5 of $[\mathrm{AAHV}]$, for every complex $\mathbf{F}$ in $\mathbb{K}(\mathcal{F}(\bmod -A))$, there is a triangle

$$
\mathbf{G} \longrightarrow \mathbf{F} \longrightarrow \mathbf{C} \rightsquigarrow
$$

in $\mathbb{K}(\mathcal{F}(\bmod -A))$ with $\mathbf{C} \in \mathbb{K}(\operatorname{Cot}-\mathcal{F}(\bmod -A))$ and $\mathbf{G} \in \mathbb{K}_{\mathrm{ac}}(\mathcal{F}(\bmod -A))$. So, there is a triangle functor

$$
\psi: \frac{\mathbb{K}(\mathcal{F}(\bmod -A))}{\mathbb{K}_{\mathrm{ac}}(\mathcal{F}(\bmod -A))} \longrightarrow \mathbb{K}(\operatorname{Cot}-\mathcal{F}(\bmod -A)),
$$

given by $\psi(\mathbf{F})=\mathbf{C}$; see $[\mathrm{M}$, Proposition 2.6] for more details.

\section{Dualities of homotopy Categories}

Throughout the section, $A$ is a right and left coherent ring. Our aim is to show that there is a duality of triangulated categories

$$
\mathbb{K}^{\mathrm{b}}\left(\bmod -A^{\mathrm{op}}\right) \longrightarrow \mathbb{K}^{\mathrm{b}}(\bmod -A),
$$

that restricts to a duality between their full subcategories of all acyclic complexes

$$
\mathbb{K}_{\mathrm{ac}}^{\mathrm{b}}\left(\bmod -A^{\mathrm{op}}\right) \longrightarrow \mathbb{K}_{\mathrm{ac}}^{\mathrm{b}}(\bmod -A) .
$$

3.1. In view of $2.3,2.4$ and 2.5 , we get an equivalence $\Psi: \mathbb{D}(\operatorname{Mod}-(\bmod -A)) \rightarrow \mathbb{K}(\operatorname{PInj}-A)$ of triangulated categories, given by the following composition

$$
\Psi: \mathbb{D}(\operatorname{Mod}-(\bmod -A)) \stackrel{\varphi}{\longrightarrow} \frac{\mathbb{K}(\mathcal{F}(\bmod -A))}{\mathbb{K}_{\mathrm{ac}}(\mathcal{F}(\bmod -A))} \stackrel{\psi}{\longrightarrow} \mathbb{K}(\operatorname{Cot}-\mathcal{F}(\bmod -A)) \stackrel{\mathbb{K}(U)^{-1}}{\longrightarrow} \mathbb{K}(\operatorname{PInj}-A),
$$

of equivalences. Throughout we will use this equivalence.

Lemma 3.2. There is an equivalence

$$
\mu: \mathbb{D}\left(\operatorname{Mod}-\left(\bmod -A^{\mathrm{op}}\right)\right) \stackrel{\sim}{\longrightarrow} \mathbb{D}\left(\operatorname{Mod}-(\bmod -A)^{\mathrm{op}}\right)
$$

of triangulated categories, given by

$$
\mu(\mathbf{X})=-\otimes_{A} \Psi(\mathbf{X}),
$$

where $\Psi$ is the equivalence introduced in 3.1 .

Proof. By [GJ], an object $E$ of Mod-(mod- $A)^{\text {op }}$ is injective if and only if $E \cong-\otimes_{A} M$, for some $M \in \mathrm{PInj}-A^{\mathrm{op}}$. Therefore, the full and faithful functor PInj- $A^{\mathrm{op}} \longrightarrow \operatorname{Mod}-(\bmod -A)^{\mathrm{op}}$, given by the rule $M \mapsto-\otimes_{A} M$, induces the following triangle equivalence

$$
\mathbb{K}\left(\mathrm{PInj}-A^{\mathrm{op}}\right) \simeq \mathbb{K}\left(\operatorname{Inj}-\left(\operatorname{Mod}-(\bmod -A)^{\mathrm{op}}\right)\right) .
$$

Moreover, similar to Lemma 4.8 of [K2], one can see that the canonical functor

$$
\mathbb{K}\left(\operatorname{Inj}-\left(\operatorname{Mod}-(\bmod -A)^{\text {op }}\right)\right) \longrightarrow \mathbb{D}\left(\operatorname{Mod}-(\bmod -A)^{\text {op }}\right)
$$


is an equivalence of triangulated categories. So, in view of the equivalence

$$
\Psi: \mathbb{D}\left(\operatorname{Mod}-\left(\bmod -A^{\mathrm{op}}\right)\right) \longrightarrow \mathbb{K}\left(\mathrm{PInj}-A^{\mathrm{op}}\right)
$$

of 3.1, we get the desired equivalence

$$
\mu: \mathbb{D}\left(\operatorname{Mod}-\left(\bmod -A^{\mathrm{op}}\right)\right) \stackrel{\sim}{\longrightarrow} \mathbb{D}\left(\operatorname{Mod}-(\bmod -A)^{\mathrm{op}}\right) .
$$

Lemma 3.3. Let $\mu$ be the equivalence of Lemma 3.2. Let $t: M \longrightarrow N$ be an A-homomorphism of left $A$-modules. Then $\mu((-, M))=-\otimes_{A} M$ and $\mu((-, t))=-\otimes_{A} t$.

Proof. Pick $M \in$ Mod- $A^{\text {op }}$ and consider the functor $(-, M)$ as a complex concentrated in degree zero, which is a complex of flat functors. Take an injective resolution

$$
0 \longrightarrow-\otimes_{A} M \stackrel{-\otimes_{A} \varepsilon}{\longrightarrow} \mathbf{I}_{-\otimes_{A} M}
$$

of $-\otimes_{A} M$. By the characterization of injective objects of Mod- $(\bmod -A)^{\mathrm{op}}, \mathbf{I}_{-\otimes_{A} M}$ is of the form

$$
\mathbf{I}_{-\otimes_{A} M}: \quad 0 \longrightarrow-\otimes_{A} C^{1} \stackrel{-\otimes d^{1}}{\longrightarrow}-\otimes_{A} C^{2} \stackrel{-\otimes d^{2}}{\longrightarrow}-\otimes_{A} C^{3} \longrightarrow \cdots,
$$

such that for each $i \in \mathbb{N}, C^{i}$ is pure-injective [GJ]. Let $\mathbf{F}$ be the complex

$$
0 \longrightarrow M \stackrel{\varepsilon}{\longrightarrow} C^{1} \stackrel{d^{1}}{\longrightarrow} C^{2} \stackrel{d^{2}}{\longrightarrow} \cdots
$$

which is pure-exact and $\mathbf{C}$ be the following complex of pure-injectives

$$
\mathbf{C}: 0 \longrightarrow C^{1} \stackrel{d^{1}}{\longrightarrow} C^{2} \stackrel{d^{2}}{\longrightarrow} C^{3} \longrightarrow \cdots .
$$

Hence, there exists a degree-wise split exact sequence

$$
0 \rightarrow(-, \mathbf{C}) \rightarrow(-, \mathbf{F}) \rightarrow(-, M) \rightarrow 0
$$

of complexes in Mod- $\left(\bmod -A^{\text {op }}\right)$. So, there is the following triangle in $\mathbb{K}(\mathcal{F}(\bmod -A))$

$$
(-, \mathbf{F}) \rightarrow(-, M) \rightarrow(-, \mathbf{C})[1] \rightsquigarrow
$$

with $(-, \mathbf{F}) \in \mathbb{K}_{\mathrm{ac}}(\mathcal{F}(\bmod -A))$ and $(-, \mathbf{C}) \in \mathbb{K}(\operatorname{Cot}-\mathcal{F}(\bmod -A))$. Therefore, by definition, $\Psi((-, M))=\mathbf{C}[1]$. Hence, $\mu((-, M))=-\otimes_{A} \mathbf{C}[1]$, which is quasi-isomorphic to $-\otimes_{A} M$.

Finally, natural transformation $(-, t):(-, M) \longrightarrow(-, N)$ induces the natural transformation $-\otimes_{A} t:-\otimes_{A} M \longrightarrow-\otimes_{A} N$. This, in turn, can be lifted to their injective resolutions. Hence, $\mu$ takes the morphism $(-, t)$ to the morphism

$$
-\otimes_{A} t:-\otimes_{A} M \longrightarrow-\otimes_{A} N,
$$

as it was claimed.

Consider the functor $\mathfrak{D}: \bmod -\left(\bmod -A^{\mathrm{op}}\right)^{\mathrm{op}} \longrightarrow \bmod -(\bmod -A)^{\mathrm{op}}$ given by

$$
(\mathfrak{D} F)(N)=\operatorname{Hom}\left(F, N \otimes_{A}-\right),
$$

where $F \in \bmod -\left(\bmod -A^{\text {op }}\right)^{\text {op }}$ and $N \in \bmod -A$. This is a duality first considered by Auslander [A3] and then, independently, proved by Gruson and Jensen [GJ]. It is known as the Auslander-Gruson-Jensen duality.

In the following, we intent to show that there is a close relationship between the equivalence $\mu: \mathbb{D}\left(\operatorname{Mod}-\left(\bmod -A^{\mathrm{op}}\right)\right) \longrightarrow \mathbb{D}\left(\operatorname{Mod}-(\bmod -A)^{\mathrm{op}}\right)$ in Lemma 3.2 and the Auslander-GrusonJensen duality $\mathfrak{D}$. 
Proposition 3.4. There exists a fully faithful contravariant functor

$$
\zeta: \bmod -\left(\bmod -A^{\text {op }}\right)^{\text {op }} \longrightarrow \mathbb{D}\left(\operatorname{Mod}-\left(\bmod -A^{\text {op }}\right)\right)
$$

that commutes the following diagram

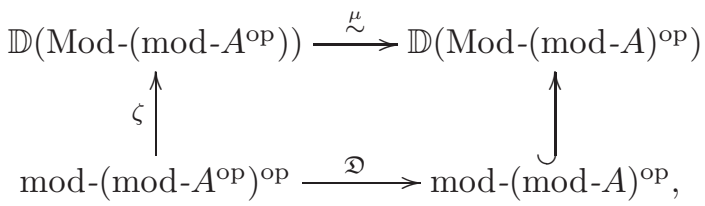

where $\mathfrak{D}$ is the Auslander-Gruson-Jensen duality.

Proof. Let $F$ be an object of mod-(mod- $\left.A^{\mathrm{op}}\right)^{\mathrm{op}}$. By definition there is an exact sequence

$$
(\dagger) \quad 0 \longrightarrow\left(M_{2},-\right) \stackrel{\left(d_{2,-}\right)}{\longrightarrow}\left(M_{1},-\right) \stackrel{\left(d_{1},-\right)}{\longrightarrow}\left(M_{0},-\right) \longrightarrow F \longrightarrow 0
$$

with $M_{i} \in \bmod -A^{\text {op }}$, for $i \in\{0,1,2\}$.

We define $\zeta(F)$ to be the complex

$$
\cdots \longrightarrow 0 \begin{array}{ccc}
\operatorname{deg} 0 & \operatorname{deg} 1 & \operatorname{deg} 2 \\
& \left(-, M_{0}\right) \stackrel{\left(-, d_{1}\right)}{\longrightarrow}\left(-, M_{1}\right) \stackrel{\left(-, d_{2}\right)}{\longrightarrow}\left(-, M_{2}\right) \longrightarrow 0 \longrightarrow \cdots .
\end{array}
$$

Note that $\zeta(F)$ is a complex of projectives and one can easily check that $\zeta$ is a full and faithful functor.

Now, we compute the image of $\zeta(F)$ under the functor $\mu$. First we show that $\mu$ maps the complex

$$
\theta: \cdots \longrightarrow 0 \longrightarrow\left(-, M_{1}\right) \stackrel{\operatorname{deg} 1}{\longrightarrow}\left(-, M_{2}\right) \longrightarrow 0 \longrightarrow \cdots
$$

in $\mathbb{D}\left(\operatorname{Mod}-\left(\bmod -A^{\text {op }}\right)\right)$ to the complex

$$
\cdots \longrightarrow 0 \longrightarrow-\otimes_{A} M_{1} \stackrel{-\otimes d_{2}}{\longrightarrow}-\otimes M_{2} \longrightarrow 0 \longrightarrow \cdots
$$

in $\mathbb{D}\left(\operatorname{Mod}-(\bmod -A)^{\text {op }}\right)$. The complex $\theta$ is the mapping cone of the following morphisms of complexes

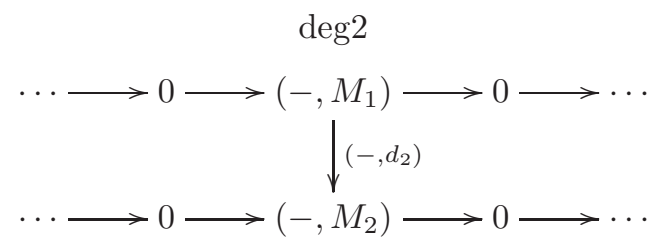

Since $\mu$ is a triangle functor, $\mu(\theta)$ is the mapping cone of the morphism

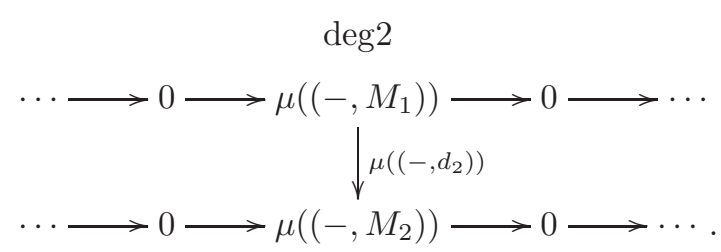


By Lemma 3.3 , the above diagram is isomorphic in $\mathbb{D}\left(\operatorname{Mod}-(\bmod -A)^{\text {op }}\right)$ to the diagram

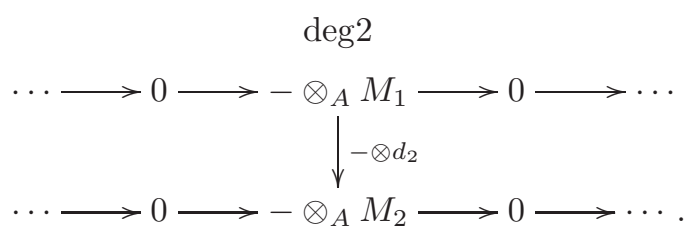

Thus, $\mu(\theta)$ is the complex

$$
\cdots \longrightarrow 0 \longrightarrow-\otimes_{A} M_{1} \stackrel{-\otimes d_{2}}{\longrightarrow}-\otimes_{A} M_{2} \longrightarrow 0 \longrightarrow \cdots
$$

with $-\otimes_{A} M_{1}$ at the 1-th position.

Now, $\zeta(F)$ is the mapping cone of the following morphism of complexes

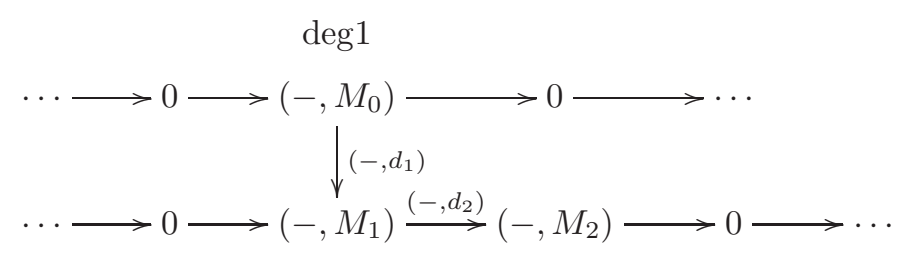

Hence, the same argument as above, applying this time to the above diagram, implies that $\mu(\zeta(F))$ is isomorphic in $\mathbb{D}\left(\operatorname{Mod}-(\bmod -A)^{\mathrm{op}}\right)$ to the complex

$$
\cdots \longrightarrow 0 \longrightarrow-\otimes_{A} M_{0} \stackrel{-\otimes d_{1}}{\longrightarrow}-\otimes_{A} M_{1} \stackrel{\operatorname{deg} 1}{\longrightarrow}-\otimes_{A} M_{2} \longrightarrow 0 \longrightarrow \cdots .
$$

Now, by applying $\mathfrak{D}$ to the exact sequence $(\dagger)$, we have the following exact sequence

$$
0 \longrightarrow \mathfrak{D} F \longrightarrow-\otimes_{A} M_{0} \stackrel{-\otimes d_{1}}{\longrightarrow}-\otimes_{A} M_{1} \stackrel{-\otimes d_{2}}{\longrightarrow}-\otimes_{A} M_{2} \longrightarrow 0 .
$$

Hence, if we consider $\mathfrak{D} F$ as a complex concentrated in degree zero, then $\mathfrak{D} F$ is quasiisomorphic to $\mu(\zeta(F))$ in $\mathbb{D}\left(\operatorname{Mod}-(\bmod -A)^{\text {op }}\right)$. This completes the proof.

Remark 3.5. It is known that for any ring $A$, the derived category $\mathbb{D}(\operatorname{Mod}-A)$ is compactly generated. Moreover, the inclusion $\operatorname{prj}-A \longrightarrow \operatorname{Mod}-A$ induces an equivalence between $\mathbb{K}^{\mathrm{b}}(\operatorname{prj}-A)$ and the full subcategory $\mathbb{D}(\operatorname{Mod}-A)^{\mathrm{c}}$ of $\mathbb{D}(\operatorname{Mod}-A)$ consisting of all compact objects $[\mathrm{Ke}]$. The same argument as in the ring case, implies that both $\mathbb{D}\left(\operatorname{Mod}-\left(\bmod -A^{\text {op }}\right)\right)$ and $\mathbb{D}\left(\operatorname{Mod}-(\bmod -A)^{\mathrm{op}}\right)$ are compactly generated and

$$
\begin{aligned}
& \mathbb{D}\left(\operatorname{Mod}-\left(\bmod -A^{\mathrm{op}}\right)\right)^{\mathrm{c}} \simeq \mathbb{K}^{\mathrm{b}}\left(\operatorname{prj}-\left(\operatorname{Mod}-\left(\bmod -A^{\mathrm{op}}\right)\right)\right), \text { and } \\
& \mathbb{D}\left(\operatorname{Mod}-(\bmod -A)^{\mathrm{op}}\right)^{\mathrm{c}} \simeq \mathbb{K}^{\mathrm{b}}\left(\operatorname{prj}-\left(\operatorname{Mod}-(\bmod -A)^{\mathrm{op}}\right)\right) .
\end{aligned}
$$

Theorem 3.6. Let $A$ be a right and left coherent ring. There is the following duality of triangulated categories

$$
\phi: \mathbb{K}^{\mathrm{b}}\left(\bmod -A^{\mathrm{op}}\right) \longrightarrow \mathbb{K}^{\mathrm{b}}(\bmod -A) .
$$

Proof. Since $\mu$ preserves direct sums, it preserves compact objects. So, by Remark 3.5, it induces the equivalence

$$
\mu \mid: \mathbb{K}^{\mathrm{b}}\left(\operatorname{prj}-\left(\operatorname{Mod}-\left(\bmod -A^{\mathrm{op}}\right)\right)\right) \longrightarrow \mathbb{K}^{\mathrm{b}}\left(\operatorname{prj}-\left(\operatorname{Mod}-(\bmod -A)^{\mathrm{op}}\right)\right)
$$


of triangulated categories. Moreover, Yoneda functors $u: \bmod -A^{\mathrm{op}} \longrightarrow \operatorname{Mod}-\left(\bmod -A^{\mathrm{op}}\right)$ and $v: \bmod -A \longrightarrow \operatorname{Mod}-(\bmod -A)^{\mathrm{op}}$ yield the following equivalences of triangulated categories

$$
\begin{aligned}
& \bar{u}: \mathbb{K}^{\mathrm{b}}\left(\bmod -A^{\mathrm{op}}\right) \longrightarrow \mathbb{K}^{\mathrm{b}}\left(\operatorname{prj}-\left(\operatorname{Mod}-\left(\bmod -A^{\mathrm{op}}\right)\right)\right), \text { and } \\
& \bar{v}: \mathbb{K}^{\mathrm{b}}(\bmod -A)^{\mathrm{op}} \longrightarrow \mathbb{K}^{\mathrm{b}}\left(\operatorname{prj}-\left(\operatorname{Mod}-(\bmod -A)^{\mathrm{op}}\right)\right) .
\end{aligned}
$$

Consequently, we have the following commutative diagram whose rows are triangle equivalences

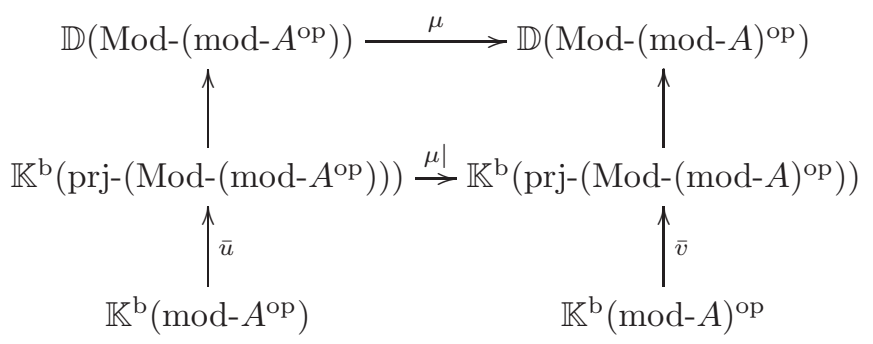

Therefore we get a duality $\phi: \mathbb{K}^{\mathrm{b}}\left(\bmod -A^{\mathrm{op}}\right) \longrightarrow \mathbb{K}^{\mathrm{b}}(\bmod -A)$, as desired.

Corollary 3.7. There is the following duality of triangulated categories

$$
\bar{\phi}: \frac{\mathbb{K}^{\mathrm{b}}\left(\bmod -A^{\mathrm{op}}\right)}{\mathbb{K}^{\mathrm{b}}\left(\operatorname{prj}-A^{\mathrm{op}}\right)} \longrightarrow \frac{\mathbb{K}^{\mathrm{b}}(\bmod -A)}{\mathbb{K}^{\mathrm{b}}(\operatorname{prj}-A)} .
$$

Proof. First we claim that the equivalence $\phi: \mathbb{K}^{\mathrm{b}}\left(\bmod -A^{\mathrm{op}}\right) \longrightarrow \mathbb{K}^{\mathrm{b}}(\bmod -A)^{\mathrm{op}}$ can be restricted to the equivalence $\phi \mid: \mathbb{K}^{\mathrm{b}}\left(\operatorname{prj}-A^{\mathrm{op}}\right) \longrightarrow \mathbb{K}^{\mathrm{b}}(\operatorname{prj}-A)^{\mathrm{op}}$. Indeed, let $P$ be a finitely generated projective left $A$-module. Then by Lemma 3.3, $\mu((-, P))=\left(-\otimes_{A} P\right)$ and $\left(-\otimes_{A} P\right) \cong\left(P^{*},-\right)$. Hence, $(\phi \mid)(P)=P^{*}$ and so belongs to $\mathbb{K}^{\mathrm{b}}(\operatorname{prj}-A)$. So, using an induction argument on the length of the complexes of $\mathbb{K}^{\mathrm{b}}\left(\operatorname{prj}-A^{\mathrm{op}}\right)$ one can deduce that, the functor $\phi$ takes any bounded complex over $\operatorname{prj}-A^{\text {op }}$ to a bounded complex over $\operatorname{prj}-A$. So, there is the following commutative diagram

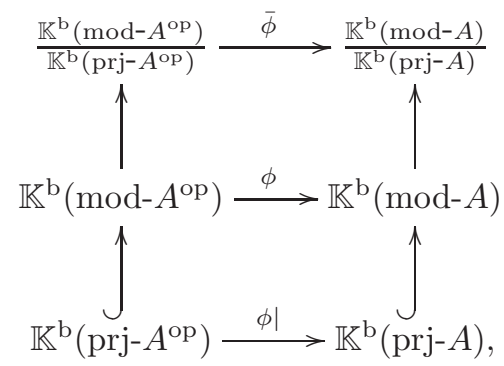

which implies the desired duality.

Remark 3.8. Let $(S, \mathfrak{m})$ be a commutative local complete Gorenstein $k$-algebra, where $k$ is an algebraically closed field. Let $M_{0}=S, M_{1}, \ldots, M_{t}$ be pairwise non-isomorphic indecomposable maximal Cohen-Macaulay $S$-modules. Set $T:=\operatorname{End}_{S}\left(\bigoplus_{i=0}^{t} M_{i}\right)$. There is a fully faithful triangle functor $\mathbb{K}^{\mathrm{b}}(\operatorname{prj}-S) \longrightarrow \mathbb{D}^{\mathrm{b}}(\bmod -T)$. By $[\mathrm{KY}$, Definition 1.1], the Verdier quotient

$$
\frac{\mathbb{D}^{\mathrm{b}}(\bmod -T)}{\mathbb{K}^{\mathrm{b}}(\operatorname{prj}-S)}
$$


is called the relative singularity category, denoted by $\Delta_{S}(T)$. This category recently has been studied in deep in [KY]. We remark that, if $S$ is a self-injective algebra, then $\Delta_{S}(T)$ is equivalent to the quotient $\frac{\mathbb{K}^{\mathrm{b}}(\bmod -S)}{\mathbb{K}^{\mathrm{b}}\left(\operatorname{prj}^{-S}\right)}$. To see this, note that in this case all modules in mod- $S$ are maximal Cohen-Macaulay, and so $T$ is the usual Auslander algebra of $S$, which is of finite global dimension, in fact, less than or equal to 2 .

\section{Artin Algebras}

In this section, we show that if $\Lambda$ is an artin algebra of finite global dimension, then the duality introduced in Theorem 3.6, restricts to a duality between their subcategories of acyclic complexes, $\mathbb{K}_{\mathrm{ac}}^{\mathrm{b}}\left(\bmod -\Lambda^{\mathrm{op}}\right)$ and $\mathbb{K}_{\mathrm{ac}}^{\mathrm{b}}(\bmod -\Lambda)$.

Throughout $\Lambda$ is an artin $R$-algebra, where $R$ is a commutative artinian ring. We need some preparations.

Lemma 4.1. Let $\gamma: \mathbb{K}_{\mathrm{ac}}^{\mathrm{b}}(\bmod -\Lambda) \longrightarrow \frac{\mathbb{K}^{\mathrm{b}}(\bmod -\Lambda)}{\mathbb{K}^{\mathrm{b}}(\operatorname{prj}-\Lambda)}$ be the triangle functor taking every complex to itself. Then $\gamma$ is full and faithful. Furthermore, $\gamma$ is dense, and so is an equivalence, if and only if $\Lambda$ has finite global dimension.

Proof. Let $f: \mathbf{X} \longrightarrow \mathbf{Y}$ be a morphism in $\mathbb{K}_{\mathrm{ac}}^{\mathrm{b}}(\bmod -\Lambda)$ such that $\gamma(f)$ vanishes in $\frac{\mathbb{K}^{\mathrm{b}}(\bmod -\Lambda)}{\mathbb{K}^{\mathrm{b}}(\operatorname{prj}-\Lambda)}$. So, there is a complex $\mathbf{Z} \in \mathbb{K}^{\mathrm{b}}(\bmod -\Lambda)$ together with a morphism $s: \mathbf{Z} \longrightarrow \mathbf{X}$ such that cone $(s) \in \mathbb{K}^{\mathrm{b}}(\operatorname{prj}-\Lambda)$ and $f \circ s$ is null homotopic. Hence, there is a morphism $t: \operatorname{cone}(s) \longrightarrow \mathbf{Y}$ making the following diagram commutative

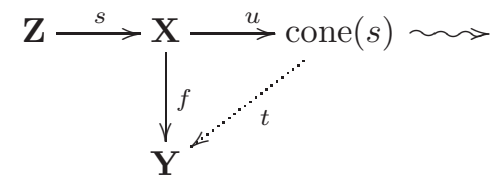

Since cone $(s) \in \mathbb{K}^{\mathrm{b}}(\operatorname{prj}-\Lambda)$ and $\mathbf{Y}$ is an acyclic complex, one can deduce that $t$, and so $f$, is null homotopic. Hence, $\gamma$ is faithful. Assume that $\mathbf{X} \stackrel{s}{\leftarrow} \mathbf{Z} \stackrel{f}{\rightarrow} \mathbf{Y}$ is a roof in $\frac{\mathbb{K}^{\mathrm{b}}(\bmod -\Lambda)}{\mathbb{K}^{\mathrm{b}}(\operatorname{prj}-\Lambda)}$ with $\mathbf{X}$ and $\mathbf{Y}$ in $\mathbb{K}_{\mathrm{ac}}^{\mathrm{b}}(\bmod -\Lambda)$. Consider a triangle $\mathbf{Z} \stackrel{s}{\rightarrow} \mathbf{X} \stackrel{u}{\rightarrow}$ cone $(s) \rightsquigarrow$ and then the composition

$$
\operatorname{cone}(s)[-1] \stackrel{u[-1]}{\rightarrow} \mathbf{Z} \stackrel{f}{\rightarrow} \mathbf{Y} .
$$

Since cone $(s) \in \mathbb{K}^{\mathrm{b}}(\operatorname{prj}-\Lambda)$ and $\mathbf{Y}$ is acyclic, the above morphism is null homotopic. So, there is a morphism $t: \mathbf{X} \longrightarrow \mathbf{Y}$ making the following diagram commutative

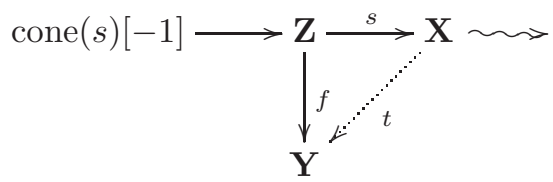


Now, the following commutative diagram implies that the roof $f \circ s^{-1}$ is equivalent to a roof $t \circ \mathrm{id}^{-1}$ in $\frac{\mathbb{K}^{\mathrm{b}}(\bmod -\Lambda)}{\mathbb{K}^{\mathrm{b}}(\operatorname{prj}-\Lambda)}$

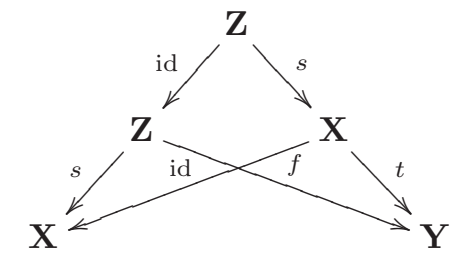

This means that $\gamma$ is full.

For the last part of the statement, let $\Lambda$ has finite global dimension and $M$ be a finitely presented $\Lambda$-module. Take a finite projective resolution $\mathbf{P}_{M} \stackrel{\pi}{\longrightarrow} M$ of $M$. The mapping cone $\operatorname{cone}(\pi)$ belongs to $\mathbb{K}_{\mathrm{ac}}^{\mathrm{b}}(\bmod -\Lambda)$ and is isomorphic to $M$ in $\frac{\mathbb{K}^{\mathrm{b}}(\bmod -\Lambda)}{\mathbb{K}^{\mathrm{b}}(\operatorname{prj}-\Lambda)}$. Therefore, $\gamma$ is dense and hence is an equivalence of triangulated categories.

For the converse, let $S$ be a simple $\Lambda$-module. Since $\gamma$ is dense, there is a bounded acyclic complex $\mathbf{X} \in \mathbb{K}_{\mathrm{ac}}^{\mathrm{b}}(\bmod -\Lambda)$ which is isomorphic to $S$ in $\frac{\mathbb{K}^{\mathrm{b}}(\bmod -\Lambda)}{\mathbb{K}^{\mathrm{b}}(\operatorname{prj}-\Lambda)}$. Hence, we have a roof $S \stackrel{s}{\longleftarrow} \mathbf{Z} \stackrel{q}{\longrightarrow} \mathbf{X}$ such that cone $(s)$ and $\operatorname{cone}(q)$ belong to $\mathbb{K}^{\mathrm{b}}(\operatorname{prj}-\Lambda)$. As $\mathbf{X}$ is an acyclic complex, a triangle $\mathbf{Z} \stackrel{q}{\rightarrow} \mathbf{X} \rightarrow \operatorname{cone}(q) \rightsquigarrow$ implies that $\mathbb{Z}$ is quasi-isomorphic to cone $(q)$. Now, consider the image of a triangle $\mathbf{Z} \stackrel{s}{\rightarrow} S \rightarrow \operatorname{cone}(s) \rightsquigarrow$ in $\mathbb{D}^{\mathrm{b}}(\bmod -\Lambda)$. Since $\mathbf{Z}$ and cone $(s)$ are isomorphic in $\mathbb{D}^{\mathrm{b}}(\bmod -\Lambda)$ to bounded complexes of finitely generated projective $\Lambda$-modules, so is $S$. This means that $S$ has finite projective dimension. The proof is now complete.

The argument in the proof of the above lemma carry over verbatim to yield the following lemma.

Lemma 4.2. Let $\gamma^{\prime}: \mathbb{K}_{\mathrm{ac}}^{\mathrm{b}}(\bmod -\Lambda) \longrightarrow \frac{\mathbb{K}^{\mathrm{b}}(\bmod -\Lambda)}{\mathbb{K}^{\mathrm{b}}(\operatorname{inj}-\Lambda)}$ be a triangle functor taking every complex to itself. Then $\gamma^{\prime}$ is full and faithful. Furthermore, $\gamma^{\prime}$ is dense, and so is an equivalence, if and only if $\Lambda$ has finite global dimension.

Proposition 4.3. Let $\Lambda$ be an artin algebra of finite global dimension. Then there is the following duality of triangulated categories

$$
\mathbb{K}_{\mathrm{ac}}^{\mathrm{b}}\left(\bmod -\Lambda^{\mathrm{op}}\right) \simeq \mathbb{K}_{\mathrm{ac}}^{\mathrm{b}}(\bmod -\Lambda) .
$$

Proof. Corollary 3.7 in conjunction with Lemma 4.1 imply the result.

Lemma 4.4. Let $\Lambda$ be an artin algebra of finite global dimension. Then there is a full and faithful functor

taking every $\Lambda$-module $M$ to itself.

$$
\lambda: \underline{\bmod }-\Lambda \longrightarrow \frac{\mathbb{K}^{\mathrm{b}}(\bmod -\Lambda)}{\mathbb{K}^{\mathrm{b}}(\operatorname{prj}-\Lambda)}
$$

Proof. By Lemma 4.1, we have the following equivalence of triangulated categories

$$
\mathbb{K}_{\mathrm{ac}}^{\mathrm{b}}(\bmod -\Lambda) \stackrel{\gamma}{\longrightarrow} \frac{\mathbb{K}^{\mathrm{b}}(\bmod -\Lambda)}{\mathbb{K}^{\mathrm{b}}(\operatorname{prj}-\Lambda)} .
$$

So, it is enough to show that the functor

$$
\underline{\bmod }-\Lambda \longrightarrow \mathbb{K}_{\mathrm{ac}}^{\mathrm{b}}(\bmod -\Lambda)
$$


mapping every module $M$ in $\underline{\text { mod}}-\Lambda$ to the mapping cone of a projective resolution $\mathbf{P}_{M} \longrightarrow M$ of $M$, is full and faithful. This follows easily from the properties of projective resolutions. Hence, the functor $\lambda$ which is the composition of the above two functors is also full and faithful.

Lemma 4.5. Let $\Lambda$ be an artin algebra of finite global dimension. Then there is a full and faithful functor

$$
\lambda^{\prime}: \overline{\bmod }-\Lambda \longrightarrow \frac{\mathbb{K}^{\mathrm{b}}(\bmod -\Lambda)}{\mathbb{K}^{\mathrm{b}}(\operatorname{inj}-\Lambda)}
$$

taking every $\Lambda$-module $M$ to itself.

Proof. The proof is similar to the proof of the above lemma. Just one should consider the functor $\varsigma: \overline{\bmod }-\Lambda \longrightarrow \mathbb{K}_{\mathrm{ac}}^{\mathrm{b}}(\bmod -\Lambda)$ which maps every module $M$ to the mapping cone of an injective resolution $M \longrightarrow \mathbf{E}_{M}$ of $M$, show that $\varsigma$ is full and faithful and then apply Lemma 4.2. We skip the details.

Let $P_{0} \stackrel{f}{\longrightarrow} P_{1} \longrightarrow M \longrightarrow 0$ be a projective presentation of the $A$-module $M$. Recall that the Auslander transpose of $M, \operatorname{Tr} M$, is defined to be the $A^{\mathrm{op}}$-module $\operatorname{Coker}\left(\operatorname{Hom}_{A}(f, A)\right)$. It is known that $\operatorname{Tr} M$ is unique up to projective equivalences and so induces an equivalence

$$
\text { Tr }: \underline{\bmod }-A^{\mathrm{op}} \longrightarrow(\underline{\bmod }-A)^{\mathrm{op}}
$$

of stable categories.

Proposition 4.6. Let $\Lambda$ be an artin algebra of finite global dimension. Then there is the following commutative diagram

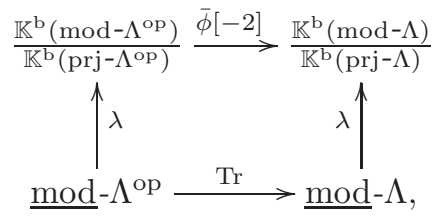

such that rows are dualities and $\lambda$ is the functor defined in Lemma 4.4.

Proof. Let $M$ be a finitely presented left $\Lambda$-module with no projective direct summands. Consider the functor $(-, M)$ in $\mathbb{D}\left(\right.$ Mod- $\left.\left(\bmod -\Lambda^{\mathrm{op}}\right)\right)$. By Lemma 3.3, $\mu((-, M))=-\otimes_{\Lambda} M$. If $Q \longrightarrow P \longrightarrow M \longrightarrow 0$ is a projective presentation of $M$, then the exact sequence

$$
0 \longrightarrow(\operatorname{Tr} M,-) \longrightarrow\left(Q^{*},-\right) \longrightarrow\left(P^{*},-\right) \longrightarrow-\otimes_{\Lambda} M \longrightarrow 0
$$

implies that $\mu((-, M))$ is isomorphic to the following complex in $\mathbb{D}\left(\operatorname{Mod}-(\bmod -\Lambda)^{\mathrm{op}}\right)$

$$
0 \longrightarrow(\operatorname{Tr} M,-) \longrightarrow\left(Q^{*},-\right) \longrightarrow\left(P^{*},-\right) \longrightarrow 0 .
$$

It follows from the definition of $\phi$, see diagram (3.1), that $\phi(M)$ is a complex

$$
0 \longrightarrow P^{*} \longrightarrow Q^{*} \longrightarrow \operatorname{Tr} M \longrightarrow 0
$$

in $\mathbb{K}^{\mathrm{b}}(\bmod -\Lambda)$. This clearly is isomorphic to $\operatorname{Tr} M[2]$ in $\frac{\mathbb{K}^{\mathrm{b}}(\bmod -\Lambda)}{\mathbb{K}^{\mathrm{b}}(\operatorname{prj}-\Lambda)}$.

Remark 4.7. It is known that if $\Lambda$ is an artin $R$-algebra, there is the duality

$$
D: \bmod -\Lambda \longrightarrow \bmod -\Lambda^{\text {op }}
$$


defined by $D(M)=\operatorname{Hom}_{R}(M, E)$, where $E$ is the injective envelope of $R / \operatorname{rad} R$. The duality $D: \bmod -\Lambda \longrightarrow \bmod -\Lambda^{\text {op }}$ can be extended to the following duality of triangulated categories

$$
\mathbb{K}^{\mathrm{b}}(\bmod -\Lambda) \stackrel{\sim}{\longrightarrow} \mathbb{K}^{\mathrm{b}}\left(\bmod -\Lambda^{\mathrm{op}}\right) .
$$

Moreover, it is known that the duality $D$ can be restricted to the duality

$$
D \mid: \operatorname{prj}-\Lambda \stackrel{\sim}{\longrightarrow} \operatorname{inj}-\Lambda^{\text {op }} .
$$

This duality also can be extended to the duality

$$
\mathbb{K}^{\mathrm{b}}(\operatorname{prj}-\Lambda) \stackrel{\sim}{\longrightarrow} \mathbb{K}^{\mathrm{b}}\left(\mathrm{inj}-\Lambda^{\mathrm{op}}\right)
$$

of triangulated categories. Therefore, we can deduce that $D$ induces a duality

$$
\frac{\mathbb{K}^{\mathrm{b}}(\bmod -\Lambda)}{\mathbb{K}^{\mathrm{b}}(\operatorname{prj}-\Lambda)} \simeq \frac{\mathbb{K}^{\mathrm{b}}\left(\bmod -\Lambda^{\mathrm{op}}\right)}{\mathbb{K}^{\mathrm{b}}\left(\operatorname{inj}-\Lambda^{\mathrm{op}}\right)}
$$

of triangulated categories, which we denote it again by $D$.

Corollary 4.8. Let $\Lambda$ be an artin algebra of finite global dimension. Then there is the following commutative diagram

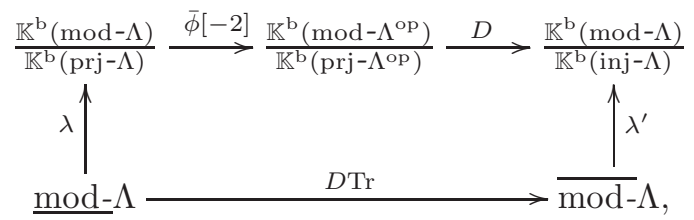

such that $\lambda$ and $\lambda^{\prime}$ are fully faithful and $D \operatorname{Tr}$ and $D \bar{\phi}[-2]$ are equivalences.

Proof. By Lemmas 4.4 and 4.5, $\lambda$ and $\lambda^{\prime}$ are fully faithful, respectively. The commutativity of the diagram follows from Proposition 4.6.

\section{SERRE FUnCtor FOR $\mathbb{K}_{\mathrm{ac}}^{\mathrm{b}}(\bmod -\Lambda)$}

In this section, we prove that if $\Lambda$ is an artin algebra of finite global dimension, then $\mathbb{K}_{\mathrm{ac}}^{\mathrm{b}}(\bmod -\Lambda)$ has Serre duality and will investigate the relationship between this Serre duality and the equivalence

$$
\frac{\mathbb{K}^{\mathrm{b}}(\bmod -\Lambda)}{\mathbb{K}^{\mathrm{b}}(\operatorname{prj}-\Lambda)} \stackrel{D \bar{\phi}[-2]}{\longrightarrow} \frac{\mathbb{K}^{\mathrm{b}}(\bmod -\Lambda)}{\mathbb{K}^{\mathrm{b}}(\operatorname{inj}-\Lambda)}
$$

of Corollary 4.8.

Recall that for a Hom-finite Krull-Schmidt $R$-linear triangulated category $\mathcal{T}$, a Serre functor is an auto-equivalence $\mathbb{S}: \mathcal{T} \longrightarrow \mathcal{T}$ such that the Serre duality formula holds, that is, we have bifunctorial isomorphisms

$$
D \mathcal{T}(X, Y) \cong \mathcal{T}(Y, \mathbb{S} X), \text { for all } X, Y \in \mathcal{T},
$$

where $D$ is the duality $\operatorname{Hom}_{R}(-, R)$.

To begin, observe that the duality

$$
D: \bmod -\Lambda \longrightarrow \bmod -\Lambda^{\mathrm{op}}
$$

induces the duality

$$
D: \bmod -(\bmod -\Lambda) \longrightarrow \bmod -(\bmod -\Lambda)^{\mathrm{op}}
$$


which maps a functor $F$ to the functor $D F(M)=D(F(M))$ for every $M \in \bmod -\Lambda$. So, every injective object in $\bmod -(\bmod -\Lambda)$ is of the form $D \operatorname{Hom}_{\Lambda}(M,-)$, for some $M \in \bmod -\Lambda$. Define a functor

$$
\mathcal{V}: \operatorname{Prj}-(\bmod -(\bmod -\Lambda)) \longrightarrow \operatorname{Inj}-(\bmod -(\bmod -\Lambda))
$$

by $\mathcal{V}\left(\operatorname{Hom}_{\Lambda}(-, M)\right)=D \operatorname{Hom}_{\Lambda}(M,-)$. It is an equivalence of categories. $\mathcal{V}$ can be naturally extended to the equivalence

$$
\mathbb{K}^{\mathrm{b}}(\operatorname{Prj}-(\bmod -(\bmod -\Lambda))) \stackrel{\sim}{\longrightarrow} \mathbb{K}^{\mathrm{b}}(\operatorname{Inj}-(\bmod -(\bmod -\Lambda)))
$$

of triangulated categories, which we denote it again by $\mathcal{V}$.

Since global dimension of $\bmod -(\bmod -\Lambda)$ is finite, $\mathcal{V}$ is in fact the equivalence

$$
\mathbb{D}^{\mathrm{b}}(\bmod -(\bmod -\Lambda)) \longrightarrow \mathbb{D}^{\mathrm{b}}(\bmod -(\bmod -\Lambda)) \text {. }
$$

Now, the same argument as in the proof of Proposition 5.3 of [ABHV] can be applied to prove that this functor is a Serre duality, i.e. for every $\mathbf{X}, \mathbf{Y} \in \mathbb{D}^{\mathrm{b}}(\bmod -(\bmod -\Lambda))$ there is the following natural isomorphism

$$
\operatorname{Hom}(\mathbf{X}, \mathbf{Y}) \cong D \operatorname{Hom}(\mathbf{Y}, \mathcal{V} \mathbf{X}),
$$

where both Hom are taken in $\mathbb{D}^{\mathrm{b}}(\bmod -(\bmod -\Lambda))$.

Proposition 5.1. Let $\Lambda$ be an artin algebra. Then $\mathbb{K}^{\mathrm{b}}(\bmod -\Lambda)$ has Serre duality.

Proof. There is an equivalence $\mathbb{D}^{\mathrm{b}}(\bmod -(\bmod -\Lambda)) \simeq \mathbb{K}^{\mathrm{b}}(\operatorname{Prj}-(\bmod -(\bmod -\Lambda)))$ of triangulated categories, because gl.dim $(\bmod -(\bmod -\Lambda))$ is finite. So, in view of Yoneda lemma, we get an equivalence

$$
\mathcal{Q}: \mathbb{K}^{\mathrm{b}}(\bmod -\Lambda) \longrightarrow \mathbb{D}^{\mathrm{b}}(\bmod -(\bmod -\Lambda))
$$

of triangulated categories. Let $\mathcal{U}: \mathbb{K}^{\mathrm{b}}(\bmod -\Lambda) \longrightarrow \mathbb{K}^{\mathrm{b}}(\bmod -\Lambda)$ be the functor that commutes the following diagram

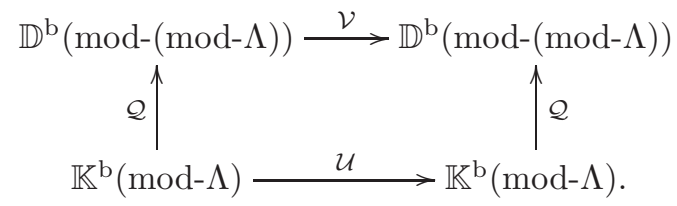

It can be easily checked that $\mathcal{U}$ is also a Serre duality functor, i.e. for every complexes $\mathbf{X}$ and $\mathbf{Y}$ in $\mathbb{K}^{\mathrm{b}}(\bmod -\Lambda)$ we have the following isomorphism

$$
\operatorname{Hom}_{\mathbb{K}^{\mathrm{b}}(\bmod -\Lambda)}(\mathbf{X}, \mathbf{Y}) \cong D \operatorname{Hom}_{\mathbb{K}^{\mathrm{b}}(\bmod -\Lambda)}(\mathbf{Y}, \mathcal{U} \mathbf{X}) .
$$

Remark 5.2. The above proposition was proved by Backelin and Jaramillo [BJ] using different approach. Their method is based on the construction of a $t$-structure in $\mathbb{K}^{\mathrm{b}}(\bmod -\Lambda)$. It also can be obtained from [ZH, Theorem 3.4]. The proof presented here uses functor category techniques.

Proposition 5.3. Let $\Lambda$ be an artin algebra of finite global dimension. Then $\mathbb{K}_{\mathrm{ac}}^{\mathrm{b}}(\bmod -\Lambda)$ has Serre duality. 
Proof. First note that the inclusion functor $i: \mathbb{K}_{\mathrm{ac}}^{\mathrm{b}}(\bmod -\Lambda) \longrightarrow \mathbb{K}^{\mathrm{b}}(\bmod -\Lambda)$ admits a right adjoint $i_{\rho}: \mathbb{K}^{\mathrm{b}}(\bmod -\Lambda) \longrightarrow \mathbb{K}_{\mathrm{ac}}^{\mathrm{b}}(\bmod -\Lambda)$. In fact, $i_{\rho}$ is defined as follows. Let $\mathbf{X}$ be a complex in $\mathbb{K}^{\mathrm{b}}(\bmod -\Lambda)$. It has a K-injective resolution $\iota_{\mathbf{X}}: \mathbf{X} \longrightarrow \mathbf{I}_{\mathbf{X}}$ with $\mathbf{I}_{\mathbf{X}} \in \mathbb{K}^{\mathrm{b}}(\operatorname{inj}-\Lambda)$. Then $i_{\rho}(\mathbf{X})=\operatorname{cone}\left(\iota_{\mathbf{X}}\right)[-1]$. We set $\mathbb{S}: \mathbb{K}_{\mathrm{ac}}^{\mathrm{b}}(\bmod -\Lambda) \longrightarrow \mathbb{K}_{\mathrm{ac}}^{\mathrm{b}}(\bmod -\Lambda)$ to be the following composition of triangle functors

$$
\mathbb{K}_{\mathrm{ac}}^{\mathrm{b}}(\bmod -\Lambda) \stackrel{i}{\longrightarrow} \mathbb{K}^{\mathrm{b}}(\bmod -\Lambda) \stackrel{\mathcal{U}}{\longrightarrow} \mathbb{K}^{\mathrm{b}}(\bmod -\Lambda) \stackrel{i_{\rho}}{\longrightarrow} \mathbb{K}_{\mathrm{ac}}^{\mathrm{b}}(\bmod -\Lambda) .
$$

For every two complexes $\mathbf{X}$ and $\mathbf{Y}$ in $\mathbb{K}_{\mathrm{ac}}^{\mathrm{b}}(\bmod -\Lambda)$, there are the following isomorphisms

$$
\begin{aligned}
\operatorname{Hom}_{\mathbb{K}_{\mathrm{ac}}^{\mathrm{b}}(\bmod -\Lambda)}(\mathbf{X}, \mathbf{Y}) & \cong \operatorname{Hom}_{\mathbb{K}^{\mathrm{b}}(\bmod -\Lambda)}(i \mathbf{X}, i \mathbf{Y}) \\
& \cong \operatorname{Hom}_{\mathbb{K}^{\mathrm{b}}(\bmod -\Lambda)}(i \mathbf{Y}, \mathcal{U} i \mathbf{X}) \\
& \cong \operatorname{Hom}_{\mathbb{K}_{\mathrm{ac}}^{\mathrm{b}}(\bmod -\Lambda)}\left(\mathbf{Y}, i_{\rho} \mathcal{U} i \mathbf{X}\right) .
\end{aligned}
$$

So $\mathbb{S}: \mathbb{K}_{\mathrm{ac}}^{\mathrm{b}}(\bmod -\Lambda) \longrightarrow \mathbb{K}_{\mathrm{ac}}^{\mathrm{b}}(\bmod -\Lambda)$ is a Serre duality.

Let $\mathcal{T}$ be a Hom-finite $R$-linear Krull-Schmidt triangulated category, where $R$ is a commutative artinian ring. A triangle $X \stackrel{f}{\longrightarrow} Y \stackrel{g}{\longrightarrow} Z \stackrel{h}{\longrightarrow} X[-1]$ in $\mathcal{T}$ is an Auslander-Reiten triangle if the following conditions are satisfied

(i) $X$ and $Z$ are indecomposable.

(ii) $h \neq 0$.

(iii) If $W$ is an indecomposable object in $\mathcal{T}$, then every non-isomorphism $t: W \longrightarrow Z$ factors through $g$.

We say that $\mathcal{T}$ has Auslander-Reiten triangles, if for every indecomposable object $W$ there exist Auslander-Reiten triangles starting and ending at $W$.

Corollary 5.4. Let $\Lambda$ be an artin algebra of finite global dimension. Then the triangulated category $\mathbb{K}_{\mathrm{ac}}^{\mathrm{b}}(\bmod -\Lambda)$ has Auslander-Reiten triangles.

Proof. It follows directly from Proposition 5.3 and Theorem I.2.4 of [RV].

The last theorem of the paper establishes a tight connection between the the functor $\mathbb{S}$ and the equivalence $D \bar{\phi}[-2]$ of Corollary 4.8.

Theorem 5.5. Let $\Lambda$ be an artin algebra of finite global dimension. Then there is the following commutative diagram

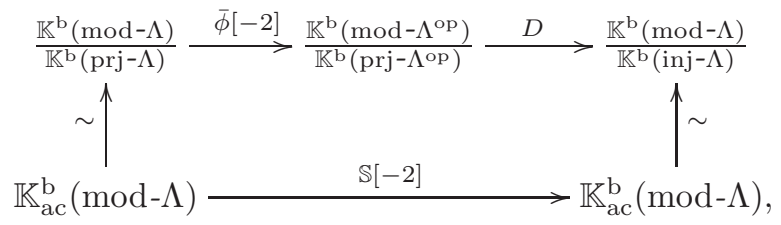

where columns are equivalences of Lemmas 4.1 and 4.2.

Proof. Let $M$ be a finitely presented $\Lambda$-module with no projective direct summands. Then $\mathcal{U}(M)$ is isomorphic to the following complex

$$
\cdots \longrightarrow 0 \longrightarrow D \operatorname{deg}-2 \quad \begin{array}{cc}
\operatorname{deg}-1 & \operatorname{deg} 0 \\
& D
\end{array} \longrightarrow P_{1}^{*} \stackrel{D f^{*}}{\longrightarrow} D P_{0}^{*} \longrightarrow 0 \longrightarrow \cdots,
$$


where $P_{1} \stackrel{f}{\longrightarrow} P_{0} \longrightarrow M \longrightarrow 0$ is the minimal projective resolution of $M$. Let

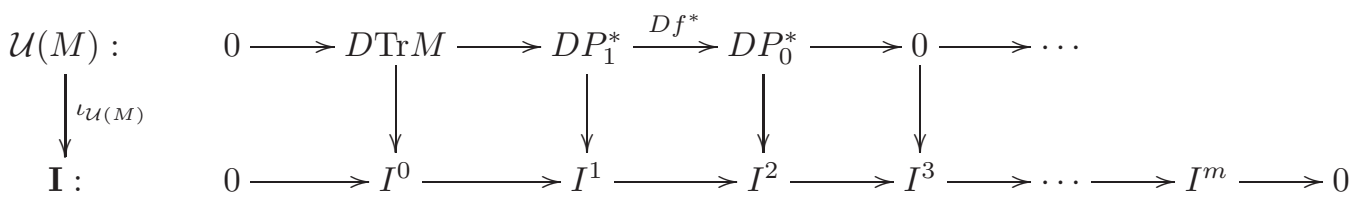

be a $\mathrm{K}$-injective resolution of $\mathcal{U}(M)$. By definition $\mathbb{S}(M)=\operatorname{cone}\left(\mathcal{L U}_{\mathcal{U}(M)}\right)[-1]$ and so it is isomorphic in $\frac{\mathbb{K}^{\mathrm{b}}(\bmod -\Lambda)}{\mathbb{K}^{\mathrm{b}}(\operatorname{inj}-\Lambda)}$ to $D \operatorname{Tr} M[2]$. Hence, $\mathbb{S}[-2](M)=D \operatorname{Tr} M$.

On the other hand by Corollary 4.8, $D(\bar{\phi}[-2])(M)=D \operatorname{Tr} M$, for every $M \in \bmod -A$. Now, an induction argument on the length of the bounded complexes in $\mathbb{K}_{\mathrm{ac}}^{\mathrm{b}}(\bmod -\Lambda)$ works to prove the commutativity of the desired diagram. See the proof of Proposition 3.4 for similar argument.

\section{REFERENCES}

[AAHV] J. Asadollahi, N. Asadollahi, R. Hafezi, R. Vahed, Auslander's Formula: Variations and Applications, Submitted.

[ABHV] J. Asadollahi, P. Bahiraei, R. Hafezi and R. Vahed, On relative derived categories, Comm. Algebra 44 (2016) 5454-5477.

[A1] M. Auslander, Coherent functors, 1966 Proc. Conf. Categorical Algebra (La Jolla, Calif., 1965) pp. 189-231 Springer, New York.

[A2] M. Auslander, Functors and morphisms determined by objects, Representation theory of algebras (Proc. Conf., Temple Univ., Philadelphia, Pa., 1976), pp. 1-244. Lecture Notes in Pure Appl. Math., Vol. 37, Dekker, New York, 1978.

[A3] M. Auslander, Isolated Singularities and Existence of Almost Split Sequences, Representation Theory II 1178 (1984), 194-241.

[BJ] E. BACKElin and O. Jaramillo, Auslander-Reiten sequences and t-structures on the homotopy category of an abelian category, J. Algebra 339 (2011), 80-96.

[BK] A. I. Bondal and M. M. Kapranov, Representable functors, Serre functors, and reconstructions, Izv. Akad. Nauk SSSR Ser. Mat. 53 (1989), no. 6, 1183-1205, 1337.

[BEIJR] D. Bravo, E. E. Enochs, A. C. Iacob, O. M. G. Jenda, J. RadA, Cotorsion pairs in $\mathbb{C}(R-M o d)$, Rocky Mountain J. Math. 42 (2012), no. 6, 1787-1802.

[GJ] L. Gruson and C. U. Jensen, Dimensions cohomologiques relièes aux foncteurs lim, Lecture Notes in Mathematics 867, Springer-Verlag, 1981, 234-294.

[H] I. Herzog, Contravariant functors on the category of finitely presented modules, Israel J. Math. 167 (2008), 347-410.

[JL] C. U. Jensen And H. Lenzing, Model theoretic algebra with particular emphasis on fields, rings, modules, Algebra Logic Appl. vol. 2. Gordon and Breach, New York (1989).

[KY] M. Kalcka And D. YAng, Relative singularity categories I: Auslander resolutions, Adv. Math., 301 (2016) 973-1021.

[Ke] B. Keller, Deriving DG categories, Ann. Sci. École Norm. Sup. (4) 27 (1994), no. 1, 63-102.

[K1] H. Krause, Approximations and adjoints in homotopy categories, Math. Ann. 353 (2012), 765-781.

[K2] H. Krause, Deriving Auslander's formula, Doc. Math. 20 (2015) 669-688.

[M] J. Mryachi, Localization of Triangulated Categories and Derived Categories, J. Algebra 141 (1991), 463-483.

[RV] I. Reiten and M. VAn den Bergh, Noetherian hereditary abelian categories satisfying Serre duality, J. Amer. Math. Soc. 15 (2002), 295-366.

[St] J. ŠŤovíčeK, Deconstructibility and the Hill lemma in Grothendieck categories, Forum Math. 25 (2013), no. 1, 193-219.

[ZH] Y. Zheng And Zh. Huang, Auslander-Reiten Triangles in Homotopy Categories, available at arxiv:1511.08964. 
Department of Mathematics, University of Isfahan, P.O.Box: 81746-73441, Isfahan, Iran

E-mail address: asadollahi@ipm.ir, asadollahi@sci.ui.ac.ir

Department of Mathematics, University of Isfahan, P.O.Box: 81746-73441, Isfahan, Iran

E-mail address: n.asadollahi@sci.ui.ac.ir

School of Mathematics, Institute for Research in Fundamental Sciences (IPM), P.O.Box: 193955746, Tehran, Iran

E-mail address: hafezi@ipm.ir

Department of Mathematics, Khansar Faculty of Mathematics and Computer Science, Khansar, IRAN

E-mail address: vahed@ipm.ir, vahed@khansar-cmc.ac.ir 